\title{
BLICKDIAGNOSE
}

Tropenmediziner aufgepasst

\section{Ein unangenehmes Reisesouvenir}

Im Rahmen einer tropenmedizinischen Sprechstunde stellt sich ein Afghane vor, welcher über die abgebildete, juckende Hautveränderung im Bereich der linken Leiste klagt. Diese sei zunächst papulös gewesen; nun stellt sie sich zentral ulzeriert bei hyperkeratotischem Randwall dar. Welche Verdachtsdiagnose haben Sie?

Der Patient hat eine kutane Leishmaniasis, für die neben o. g. Symptomen auch eine positive Reiseanamnese (endemisch im Mittelmeerraum, Nahen und Mittleren Osten, Afrika, Mittel- und Südamerika) und eine schlechte Heilungstendenz sprechen.

Die parasitäre Erregergruppe der Leishmanien wird durch Sandmücken übertragen und kann neben der kutanen Form auch zur mukokutanen oder viszeralen Leishmaniasis führen.

Der Nachweis gelingt durch einen Ulkusabstrich („slit skin smear") oder eine Gewebebiopsie. Neben lokalen Therapieoptionen (z. B. Paromomycin-Salbe, photodynamische Therapie, Kryo- und Thermotherapie) stehen bei komplizierteren Verläufen Präparate wie pentavalente Antimonate, Itraconazol, liposomales Amphotericin $B$ oder das nebenwirkungsärmere Miltefosin zur Verfügung.

Keywords leishmaniasis

Oberstabsarzt Dr. Hans-Georg Palm (1), Oberfeldarzt Uli Speck ${ }^{(1)}$,

Oberfeldarzt Dr. Guido Weisel ${ }^{(2)}$

(1) Sanitätskompanie PRT Kunduz, ISAF, Afghanistan

(2) Abteilung für Dermatologie und Venerologie, Bundeswehrkrankenhaus Ulm

Verweis auf die aktuelle Leitlinie: AWMF-online:

„S1-Leitlinie Diagnostik und Therapie der kutanen und mukokutanen Leishmaniasis in Deutschland“ JDDG Band 7, Supplement 7, Oktober 2009
Ihr besonderer Fall?

\section{Stellen Sie uns Ihren Fall vor}

Sicher sehen auch Sie ab und an einen besonders eindrucksvollen Befund in Ihrer Praxis. Fotografieren Sie ihn, schreiben Sie uns unter dem Stichwort Blickdiagnose, bei Veröffentlichung erhalten Sie einen Buchgutschein im Wert von 100 Euro.

MMW-Fortschritte der Medizin

E-Mail: reinhold.manhart@springer.com

Fax: 089/203043-31424 